\title{
First Analysis and Experiments in Aerial Manipulation Using Fully Actuated Redundant Robot Arm
}

\author{
Felix Huber, Konstantin Kondak, Kai Krieger, Dominik Sommer, Marc Schwarzbach, \\ Maximilian Laiacker, Ingo Kossyk, Sven Parusel, Sami Haddadin and Alin Albu-Schäffer
}

\begin{abstract}
In this paper we describe a system for aerial manipulation composed of a helicopter platform and a fully actuated seven Degree of Freedom (DoF) redundant industrial robotic arm. We present the first analysis of such kind of systems and show that the dynamic coupling between helicopter and arm can generate diverging oscillations with very slow frequency which we called phase circles. Based on the presented analysis, we propose a control approach for the whole system. The partial decoupling between helicopter and arm - which eliminates the phase circles - is achieved by means of special movement of robotic arm utilizing its redundant DoF. For the underlying arm control a specially designed impedance controller was proposed. In different flight experiments we showcase that the proposed kind of system type might be used in the future for practically relevant tasks. In an integrated experiment we demonstrate a basic manipulation task - impedance based grasping of an object from the environment underlaying a visual object tracking control loop.
\end{abstract}

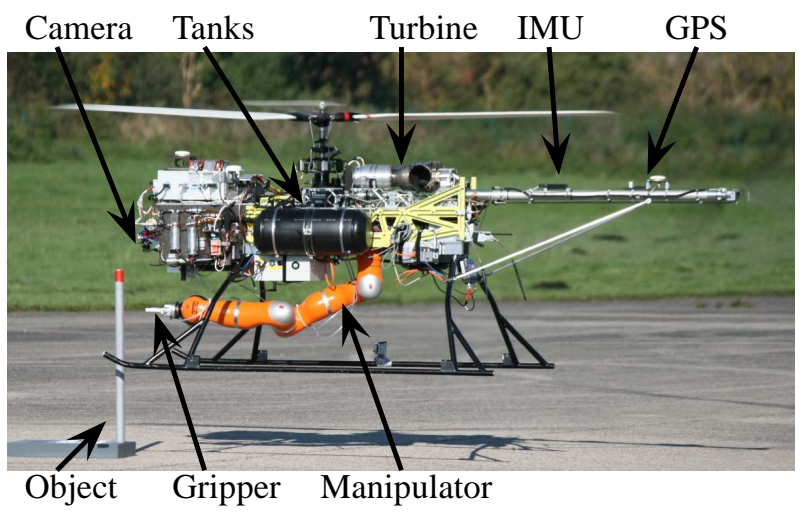

Fig. 1. First experimental platform for aerial manipulation with a 7-DoF industrial robot arm.

\section{INTRODUCTION}

\section{A. Applications, Motivation}

Unmanned Aerial Systems (UASs) have meanwhile become an important status in industrial and scientific fields. Normally mentioned in the course of standard applications like inspecting, filming, exploring, observing or in military aspects. The integration of further robotic technologies into UASs extends the range of possible applications. An UAS with an integrated robotic arm can be used for manipulation tasks involving direct physical interaction with the environment like:

All authors are with the Institute of Robotics and Mechatronics, DLR German Aerospace Center, Wessling, Germany,f.huber at dlr.de
- inspection and maintenance of industry sites (chimneys, pipelines, tanks etc.). For this operations a contact with the environment can be necessary or even unavoidable.

- constructions in uneven terrains.

- taking samples of material from areas difficult to access for scientific (geological, biological) purposes.

- taking samples of material (soil, leaves) for forestry (pest infestation of trees) and agriculture (control and optimization of fertilizer use) purposes.

- catastrophic scenarios, e.g. building up basic infrastructure in disaster areas. Equipment like antennas for communication or not drop-able first aid supply could be installed by UASs.

- a test platform for simulating on-orbit servicing operations.

Progress in that field of research was already shown by a few authors [1] - [8]. In most of these interesting concepts a simple manipulation device with a low-complexity gripper was used. In this work, we address the task of manipulation by an UAS with a seven DoF redundant robot arm (Fig.1). According to our experience in robotic manipulation, even for simple tasks a fully actuated manipulator is needed. As we show in this paper, an additional degree of freedom can be used for minimizing the influence of the arm motion to the helicopter platform.

The paper is organized as follows. After completing the first section with a general description of the system and the expected problems in manipulation from a hovering helicopter, we pass in Section II to the mathematical description of the interaction between helicopter and arm and of the occurrence of the phase cycle phenomena. In Section III we go into some detail of the arm control especially the usage of the additional DoF for minimizing the manipulator influence to the helicopter. The setup of the experimental platform is described in Section IV. Finally we summarize results and experiences in Section V.

\section{B. General Description of the System}

Fig. 1 depicts the principle structure of the system. It is composed of three main components: a flying platform, a manipulator rigidly mounted on the fuselage and a sensor system, e.g. a vision system, for tracking the manipulation object. In this paper we consider the following general simplified mission: navigation to the specified target area using GPS, activation of the camera system for object tracking, approaching the item to perform a manipulation using data 
from the camera system, hovering very closely to the object and manipulating with the robot arm.

\section{Aspects of the Task}

To fulfill the aforementioned representative mission following aspects should be investigated:

- a precise helicopter control,

- the interaction between hovering helicopter and moving manipulator,

- a proper control for the robot arm, and

- the interaction between complete system and fixed environmental objects.

A high performance position control for hovering is necessary for the success of the manipulation task. The control of the helicopter and manipulator in the last phase can be realized by different approaches. The system can be controlled by:

- completely decoupled controllers, the arm motion is a disturbance for the helicopter controller and vice versa.

- separate controllers for the subsystems, but taking into account the their kinematic coupling.

- one controller taking into account the dynamics of the complete system.

We suppose that the third approach could provide the best performance. But in this case an exact model of the whole system, probably with online adaptation, is required. In simulation experiments we figured out that in the first approach the interaction between arm and helicopter could generate diverging oscillations with slow frequency which we called phase circles (helicopter starts to move slowly in circles with increasing radius). Therefore, we develop the system according to the second approach taking into account the kinematic coupling between arm and helicopter platform. This coupling and the arm control are described in the following sections in detail. The high precision helicopter control is presented in [9].

\section{Force INTERACTION BETWEen HOVERING HELICOPTER AND MOVING ROBOT ARM}

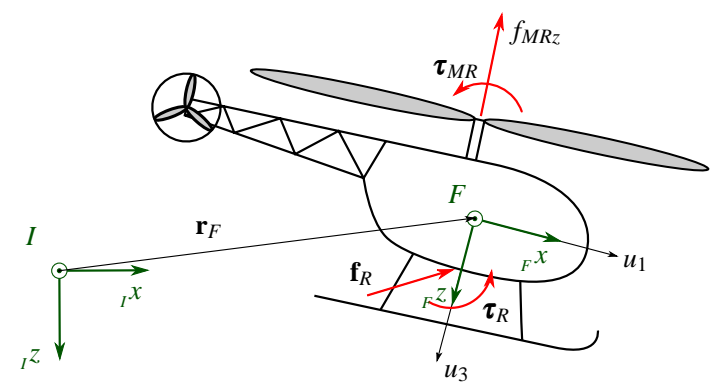

Fig. 2. Interaction between hovering helicopter and moving robot arm.

This section introduces the simplified model of a helicopter to understand the effects when a moving robot arm is mounted under the helicopter fuselage. The flying platform is composed of two rigid bodies, the fuselage and the main rotor. The second is modeled as a solid disk rotating around its vertical axis, with same mass distribution and diameter as the real rotor. The frame $F$ is fixed to the fuselage as depicted in Fig.2. We introduce six generalized velocities: $u_{1,2,3}$ for the translation of frame $F$ and $u_{4,5,6}$ for the roll, pitch and yaw rates also defined in $F$. The toques $\tau_{M R 1,2}$ and the lifting force $f_{M R 3}$ generated by the main rotor are considered as the system inputs. The equations of the translational and rotational dynamics of the helicopter can be found in more detail in [10]. With the described assumptions in [10] the rotational dynamics have the following reduced form:

$$
\begin{aligned}
& \tau_{M R 1}-\tau_{R 1}+K_{12} u_{5}+K_{11} \dot{u}_{4}=0, \\
& \tau_{M R 2}-\tau_{R 2}+K_{21} u_{4}+K_{22} \dot{u}_{5}=0 .
\end{aligned}
$$

The coefficients $K_{x x}$ depend on constant parameters and on the constant rotor speed $\omega_{M R}$. The motion around the ${ }_{F} z$ axis (in (1) and (2) not considered) is controlled mainly by the tail rotor and thus the equation for $\dot{u}_{6}$ is assumed to be decoupled. One can clearly see that (1) and (2) are coupled through $u_{4,5}$. A moving robot arm beneath the aircraft creates additional torques $\tau_{R}$ interacting with the system.

Also like in [11], considering the torques $\tau_{M R}$ and $\tau_{R}$ as constant and due to the linearity of (1) and (2) a closed form solution can be calculated :

$$
\begin{aligned}
& u_{4}=-\frac{\left(\tau_{M R 2}-\tau_{R 2}\right)}{2 \beta}+\sin (\ldots), \\
& u_{5}=\frac{\left(\tau_{M R 1}-\tau_{R 1}\right)}{2 \beta}+\cos (\ldots) .
\end{aligned}
$$

Here $\beta$ depends on the fuselage and rotor properties and its rotation speed. In (3) and (4) the rest terms on the right hand side do not contribute to the understanding of the described interaction between arm and helicopter and are neglected for sake of clarity. One can clearly see from the first part of the equations that torque applied to the roll axis generates a rotation around the perpendicular pitch axis.

This so-called gyro-effect has a serious influence on an aerial manipulation task. Assume the helicopter is hovering at the target area with the Tool Center Point (TCP) at the desired position. A displacement of the fuselage $\Delta x_{F}$ forces the robot arm to move in an extended configuration to keep its TCP position constant $\left(\Delta x_{T C P}=0\right)$, see Fig.3. This adjustment motion generates a movement $\Delta x_{C o G}$ of the Center of Gravity ( $\mathrm{CoG}$ ) of the system. The $\mathrm{CoG}$ movement $\Delta x_{C o G}$ induces a torque $\tau_{R 2}$ to the fuselage around the ${ }_{F} y$ axis. According to (3) the helicopter is rolling around its ${ }_{F} x$ axis and the thrust $f_{M R 3}$ causes a motion in $u_{2}$ direction. Continuing this principle yield the chain

$$
\Delta x_{F} \rightarrow \Delta x_{C o G} \rightarrow \tau_{R 2} \rightarrow u_{4} \rightarrow u_{2} \rightarrow \Delta y_{F} \rightarrow \Delta y_{C o G} \rightarrow \ldots
$$

This system behavior can be described with the scheme in Fig.4 for more details refer to [10]. Without blocks $P_{1}^{*}$ and $P_{2}^{*}$ the decoupled feedback system corresponds to a helicopter with the two independently controlled directions $(x, y)$ and the outputs $y_{1}=x, y_{2}=y$. The inputs $r_{1,2}$ are the desired position values $x_{d}$ and $y_{d}$. The blocks $C_{1,2}^{t}$ and $C_{1,2}^{r}$ represent 


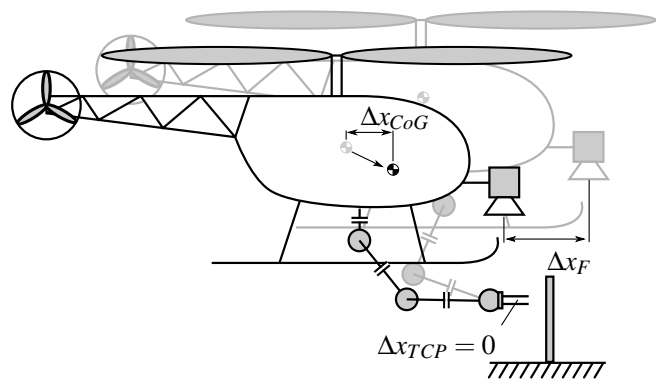

Fig. 3. Movement of the systems CoG as result of displacement compensation by the manipulator.

the controllers for the translation and rotation respectively. $P_{1}^{*}$ and $P_{2}^{*}$ imply the reaction of the helicopter due to the movement of the arm, with the output torques $\tau_{R 1}$ and $\tau_{R 2}$ acting on the fuselage. In Fig. 4 the inversion and decoupling blocks of the controller are combined with corresponding blocks in the helicopter model so the translational and rotational dynamics of the helicopter are simplified represented by integrators. In case of a symmetric system with $C_{1}^{t, r}=C_{2}^{t, r}$ the transfer function $G_{11}=y_{1} / r_{1}$ can be written as

$$
G_{11}=\frac{C^{r} C^{t} s^{3}+\left(C^{r}\right)^{2} C^{t} s^{2}+\left(C^{r} C^{t}\right)^{2}-P_{1}^{*} P_{2}^{*}}{s^{6}+2 C^{r} s^{5}+\left(C^{r}\right) s^{4}+2\left(C^{r} C^{t}\right)^{2} s^{3}+2\left(C^{r}\right)^{2} C^{t} s^{2}+\left(C^{r} C^{t}\right)^{2}-P_{1}^{*} P_{2}^{*}}
$$

The term $P_{1}^{*} P_{2}^{*}$ in the denominator has influence on the system poles. It can be shown that for practically relevant system parameters the system poles could have non-negative real part and yield low frequency oscillations of the system states. These will induce the system to move in cycles with increasing radius (phase cycles), see left picture of Fig.5. One elegant solution to avoid this effect, is to set one of the blocks $P_{1}^{*}$ or $P_{2}^{*}$ to zero, a simulation result is shown in the right graph of Fig.5. This can be realized with a restricted motion of the arm which keeps its CoG in the lateral plane of the helicopter. For this we present a capable arm controller in the next section.

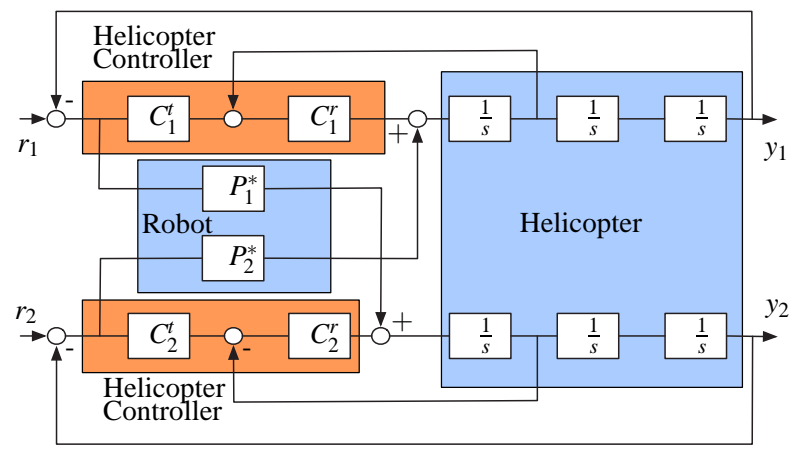

Fig. 4. Simplified scheme of helicopter with manipulator in $x$ and $y$ direction.

\section{ROBOT ARM CONTROL}

A reasonable realization of the aforementioned feature is only feasible with a redundant robot arm to be able to
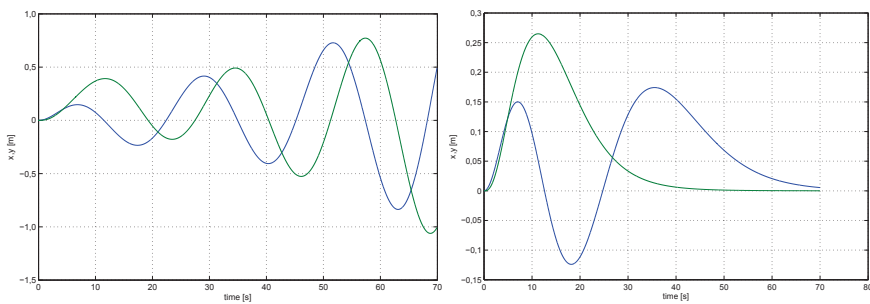

Fig. 5. Simulation results: System states $x$ and $y$ start to oscillate due to the coupling (left). Oscillations do not occur when one of the blocks $P_{1}^{*} P_{2}^{*}$ is eliminated (right).

retain all six end-effector DoFs. Therefore, we use a 7 DoF Light Weight Robot (LWR) for manipulation. Besides the good payload to weight ratio of more than 0.5 , it is also beneficial for the application that we have the possibility of measuring all seven joint torques $\left(\tau_{J, i}, i=1 \ldots 7\right)$ with built in sensors. This allows us to implement complex control strategies like impedance control, e.g. described in [12]. We think impedance control is an ideal control concept because it gives the arm an active elasticity and hence a further possibility to reduce the interaction force on the fuselage in case of a contact. Especially by an undesired collision, the arm can react with a preset stiffness and absorb a part of the impact.

For the manipulation task the motion is defined by a desired Cartesian position $\mathbf{x}_{d}$. With the impedance control and an adequate trajectory interpolation, the TCP of the arm is pulled to the desired position by a virtual spring damper system, like illustrated in Fig.6.

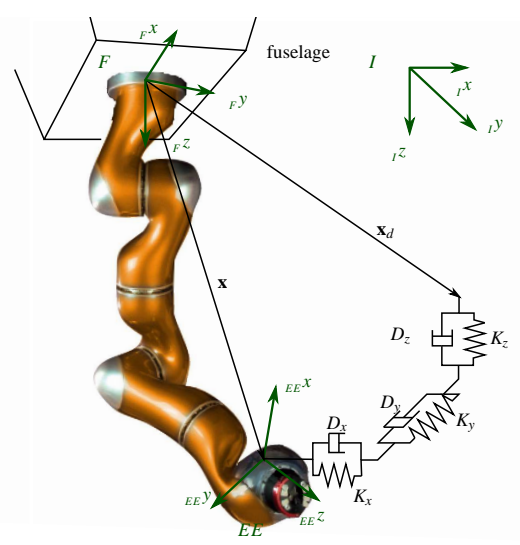

Fig. 6. Illustration of impedance control for the LWR robot arm. $\mathbf{x}$ is the actual end effector position. $D_{x, y, z}$ and $K_{x, y, z}$ are the tunable components of the virtual spring damper system.

Due to the redundancy we do not have a completely defined configuration of the robot joints and those which do not move the end-effector position have to be considered. Thus a nullspace strategy must be designed. This opens up the possibility for additional conditions e.g. the aforementioned $\mathrm{CoG}$ control. In the following this control strategy will be outlined.

The robot arm can be described by the following system 
of differential equations:

$$
\begin{aligned}
\mathbf{M}(\mathbf{q}) \ddot{\mathbf{q}}+\mathbf{C}(\mathbf{q}, \dot{\mathbf{q}}) \dot{\mathbf{q}}+\mathbf{g}(\mathbf{q}) & =\boldsymbol{\tau}_{J}+\boldsymbol{\tau}_{\text {ext }} \\
\mathbf{B} \ddot{\boldsymbol{\theta}}+\boldsymbol{\tau}_{J} & =\boldsymbol{\tau}_{m}-\boldsymbol{\tau}_{f} \\
\boldsymbol{\tau}_{J} & =\mathbf{K}(\boldsymbol{\theta}-\mathbf{q})+\mathbf{D}(\dot{\boldsymbol{\theta}}-\dot{\mathbf{q}}) .
\end{aligned}
$$

Where $\boldsymbol{\theta}$ and $\mathbf{q}$ are the motor the link side position, respectively. $\mathbf{M}(\mathbf{q}), \mathbf{G}(\mathbf{q}, \dot{\mathbf{q}}) \dot{\mathbf{q}}$ and $\mathbf{g}(\mathbf{q})$ are the link side inertia matrix, the centrifugal and Coriolis vector, and the gravity vector respectively. The inputs on the right hand side of (7) are the joint torques $\tau_{J}$ and the external torques $\boldsymbol{\tau}_{\text {ext }}$ acting on the robot arm. The joint torque $\tau_{J}$, which is primary characterized by the harmonic-drive gearboxes and the torque sensors, is modeled in (9) as a linear spring damper system with the joint stiffness matrix $\mathbf{K}=\operatorname{diag}\left(k_{i}\right)$ for the intrinsic elasticity and a damping matrix $\mathbf{D}=\operatorname{diag}\left(d_{i}\right) . \mathbf{B}$ in (8) is the motor inertia matrix and $\tau_{f}$ contains the friction torques.

The Cartesian impedance control consists of an underlying joint torque controller with the desired torque vector $\tau_{d}$ as input $\left(\boldsymbol{\tau}_{m}=\boldsymbol{\tau}_{m}\left(\boldsymbol{\tau}_{d}\right)\right)$. The desired torque is composed as

$$
\tau_{d}=\tau_{d, \text { Cart }}+\tau_{d, N S p}+\mathbf{g}(\mathbf{q})
$$

with $\tau_{d, \text { Cart }}$ as the Cartesian impedance controller torque only affecting the end-effector and $\tau_{d, N S p}$ for the manipulators nullspace motion. For the reaching task $\tau_{d, \text { Cart }}$ is designed as

$$
\tau_{d, \text { Cart }}=\mathbf{J}_{\mathbf{q}}^{T}\left(\mathbf{K}_{\mathbf{x}}\left(\mathbf{x}(\mathbf{q})-\mathbf{x}_{d}\right)+\mathbf{D}_{\mathbf{x}}\left(\dot{\mathbf{x}}(\mathbf{q})-\dot{\mathbf{x}}_{d}\right)\right)
$$

with the manipulators Jacobian $\mathbf{J}(\mathbf{q})$ with the current Cartesian end-effector position $\mathbf{x}(\mathbf{q})$ and velocity $\dot{\mathbf{x}}(\mathbf{q})$. $\mathbf{K}_{\mathbf{x}}$ and $\mathbf{D}_{\mathbf{x}}$ are positive definite matrices for the desired stiffness and damping. The desired Cartesian position $\mathbf{x}_{d}$ and velocity $\dot{\mathbf{x}}_{d}$ are provided by the visual tracking. Furthermore equation (10) includes a gravity compensation term $\mathbf{g}(\mathbf{q})$. We get the actual gravitation from the on-board attitude sensors.

The desired nullspace behavior can be described as a force $\mathbf{f}_{C o G}$ acting on the forth joint (ellbow joint) trying to pull the arm in a configuration that has the $\mathrm{CoG}$ of the manipulator as close to the lateral plane $P$ of the helicopter as possible (see Fig.7). The relationship between the force $\mathbf{f}_{C o G}$ and the torque $\tau_{C o G}$ can be written as

$$
\tau_{C o G}=\mathbf{J}_{4}^{T}(\mathbf{q}) \mathbf{f}_{C o G}
$$

with the manipulators Jacobian $\mathbf{J}_{4}(\mathbf{q})$ at the forth link.

The vector $\tau_{C o G}$ has to be projected into the nullspace in order to prevent interferences with the end-effector control. A sufficient mapping is given by

$$
\boldsymbol{\tau}_{d, N S p}=\left(\mathbf{I}-\left(\mathbf{J}(\mathbf{q})^{+} \mathbf{J}(\mathbf{q})\right)^{T}\right) \boldsymbol{\tau}_{C o G}
$$

where $\mathbf{I}$ is the identity matrix and $\mathbf{J}(\mathbf{q})^{+}$the pseudoinverse (Moore-Penrose) of the manipulators Jacobian $\mathbf{J}(\mathbf{q})$ (see [13]).

The force in (12) can be characterized by e.g. a springdamper system, therefore it is proportional to the distance $\left\|\mathbf{d}_{P, C o G}\right\|$ between the CoG $\mathbf{r}_{C o G}$ and the vertical plane $P$ and has the direction of $\mathbf{d}_{P, C o G}$

$$
\mathbf{f}_{C o G}=\mathbf{f}_{C o G}\left(\mathbf{d}_{P, C o G},\left\|\mathbf{d}_{P, C o G}\right\|\right) \text {. }
$$

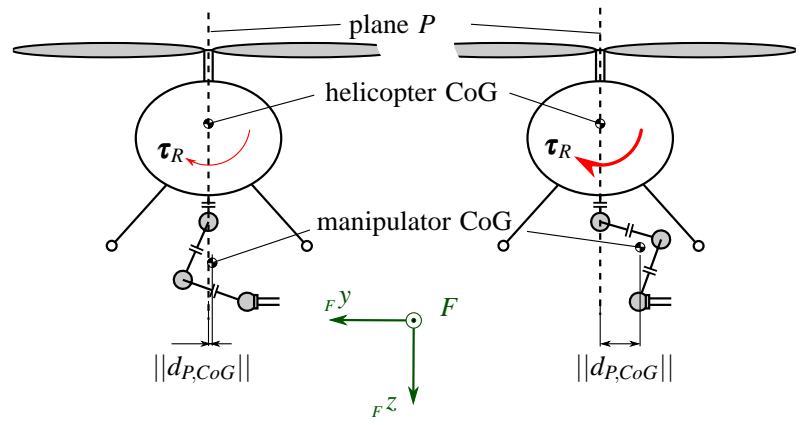

Fig. 7. Influence of different LWR configurations on helicopter fuselage. Keeping the CoG near by the plane $P$ reduces the interaction torque $\tau$. The TCP position is fixed.

The distance vector $\mathbf{d}_{P, C o G}$ is

$$
\mathbf{d}_{P, C o G}=\frac{\mathbf{n}_{d e s}^{T} \mathbf{r}_{C o G}-\mathbf{n}_{d e s}^{T} \mathbf{p}}{\|\mathbf{n}\|} \mathbf{n}_{d e s}
$$

with the normal $\mathbf{n}_{\text {des }}$ and an arbitrary point $\mathbf{p}$ in the plane $P$. $\mathbf{f}_{C o G}$ tries to keep the $\mathrm{CoG}$ as close to the plane $P$ as possible.

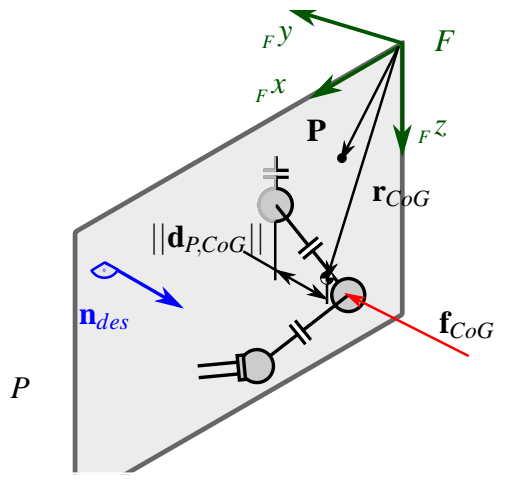

Fig. 8. Ilustration for equations (14) and (15).

To avoid collisions of the gripper with the skid elements and to prevent the robot arm TCP to be beyond the skid plane, the arm was caged in 'virtual walls' to keep it in a good working position (see Fig.9). A virtual force $\mathbf{f}_{V W}$, with repelling character, is created when the robot arm attempts to move beyond these walls. This force acting at the TCP is mapped into the joint space similar to (12):

$$
\boldsymbol{\tau}_{V W}=\mathbf{J}^{T}(\mathbf{q}) \mathbf{f}_{V W} .
$$

Equation (16) has to be added to (10) to get the final control law. In the rest of the paper we give a short overview of the first experiments.

\section{EXPERIMENTAL PlatForm}

The helicopter we use for our first tests is an experimental platform provided by the company SWISS UAV with a classical setup composed of a main and a tail rotor. Both rotors of this platform are driven by a turbine with a rated power of $25 \mathrm{~kW}$. The mounting point of the arm allows the trim of the entire system as can be seen in Fig.1. 


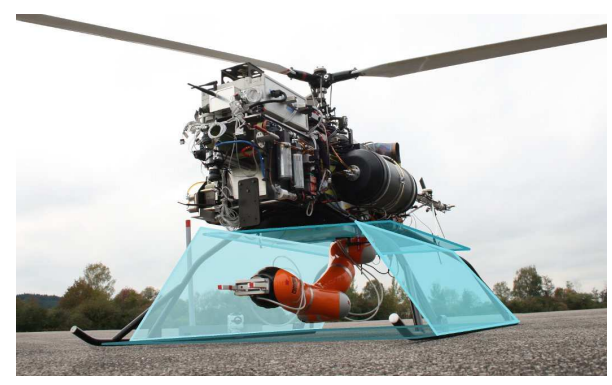

Fig. 9. Helicopter/ Manipulator system with illustrated virtual walls, to avoid self-collision.

Operating the end-effector beneath the skid plane is avoided due to safety reasons. Due to that, the working space was restricted to a cubic area of about $300 \mathrm{~mm}$ length, $300 \mathrm{~mm}$ width and $200 \mathrm{~mm}$ height. The maximal allowed Cartesian velocity of the end effector is $\dot{\mathbf{x}}_{\max }=1 \mathrm{~m} / \mathrm{s}$ and its maximum acceleration $\ddot{\mathbf{x}}_{\max }=10 \mathrm{~m} / \mathrm{s}^{2}$. The robot arm is equipped with a pneumatic gripper. Its air pressure tanks are fixed at the fuselage. For the robot arm control the authors use the Robot Control Unit (RCU) framework [14] which was successfully tested in many experiments at German Aerospace Center (DLR). The control scheme used in the first experiments is shown in Fig.10.

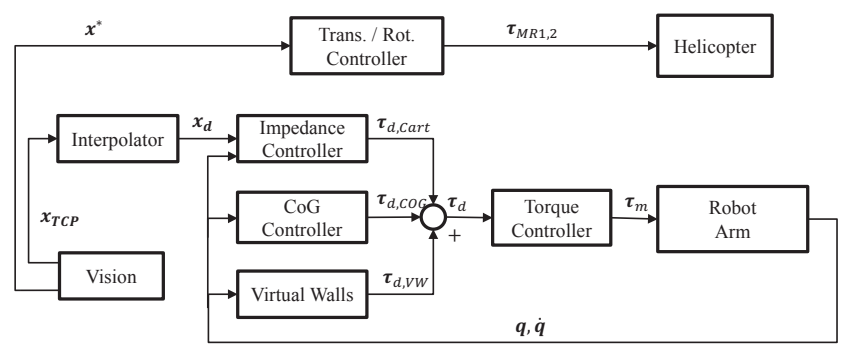

Fig. 10. Scheme of control structure

\section{EXPERIMENTS}

The experimental work was devoted to the following two subjects: investigation of a principal possibility to operate the proposed system and investigation of possibility to perform simple manipulation tasks.

Helicopters are generally difficult to operate due to oscillation effects caused by the main rotor, e.g. mechanical resonance in the air and ground resonance. A serial manipulator connected to the helicopter fuselage makes the system extremely sensitive to all vibration and resonance effects. In the very first step we had to verify mechanical stability of the system, possibility to avoid resonance during the seed-up of the rotor and during the flight. After that, the resistance of the arm electronics was successfully verified.

In the third step we verified the calculated influence of the arm movement on the helicopter in hovering. Due to our calculations, the static (very slow) displacement of the CoG caused by arm movement in the defined workspace, should have only a small influence on the helicopter. This influence could be compensated by helicopter controller as a disturbance without taking the arm into account. For that following flight tests were conducted: the helicopter was hovering in position control mode, the TCP of the arm was moved between extreme positions in the workspace with different speeds. The results were as expected. The influence of dynamics during the arm movement within specified range, see Sec.IV, is negligible. An indirect confirmation for existence of phase circles we got from the following experiment: the helicopter was hovering in manual control mode and the arm was commanded to move the TCP from one extreme position in the workspace to the other one. After the arm started to move, the safety pilot did not change the position of control sticks for couple of seconds in order to allow the helicopter to generate natural response to the arm motion. An example of this natural response is shown in Fig.11 where the angular speeds for roll $\dot{u}_{4}$ and pitch $\dot{u}_{5}$ are shown. The rectangle signal in Fig.11 is the position of sixth arm joint $q_{6}$ and denotes the start and end of arm movement. In Fig. 11 the reaction of the fuselage in roll and pitch axes to the arm movement can be seen (marked with red circles in the Fig.11). This corresponds to the behavior described by (4) and (3) and to the causal chain given by (5) which can generate phase circles described in Sec.II.

To verify the possibility to perform a simple manipulation task a general simplified mission described in Sec.I-B was executed.

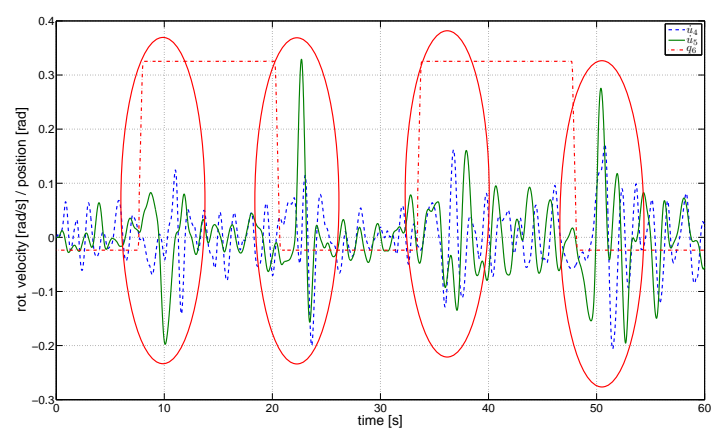

Fig. 11. Roll $\dot{u}_{4}$ and pitch $\dot{u}_{5}$ rates measured during arm movement (only $q_{6}$ is plotted).

After a take-off, the helicopter approached the target area by GPS way-points. During this flight the arm was parked in a safe configuration. After arrival at manipulation area, the vision system was activated for exact localization of the object. After its activation, the arm started the manipulation while compensating the movement of the helicopter. After fulfilling the manipulation successfully, the arm was folded in its safe park configuration and the platform returned to home base.

Snapshots of this experiment are shown in Fig.12 and 13 from different points of view. The proposed aerial manipulation system was able to grasp the pole and to pull it out of the fixing. The video of the experiment can be seen in the supplementary multimedia material of this paper. 
The defined limits for the dynamics of the arm in Sec.IV allowed the compensation of the helicopter movement caused by the wind gusts and ground effect. The experiments were conducted by good weather conditions, wind speed under 4 $\mathrm{m} / \mathrm{s}$.

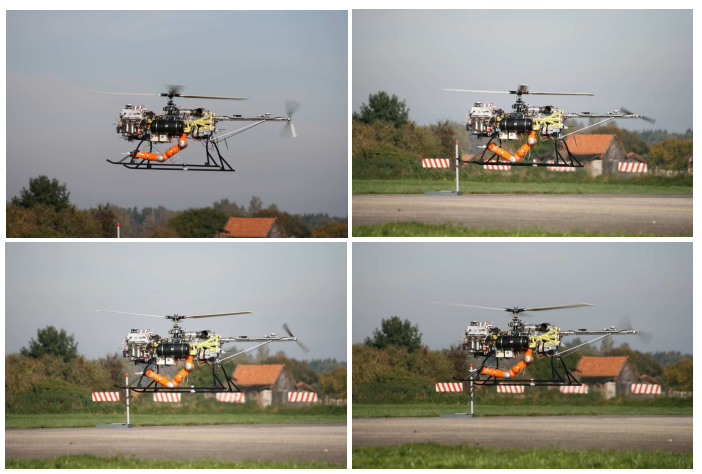

Fig. 12. Snapshots of manipulation task. Helicopter is arriving at the target area, hovering with activated vision system and grasping the pole.

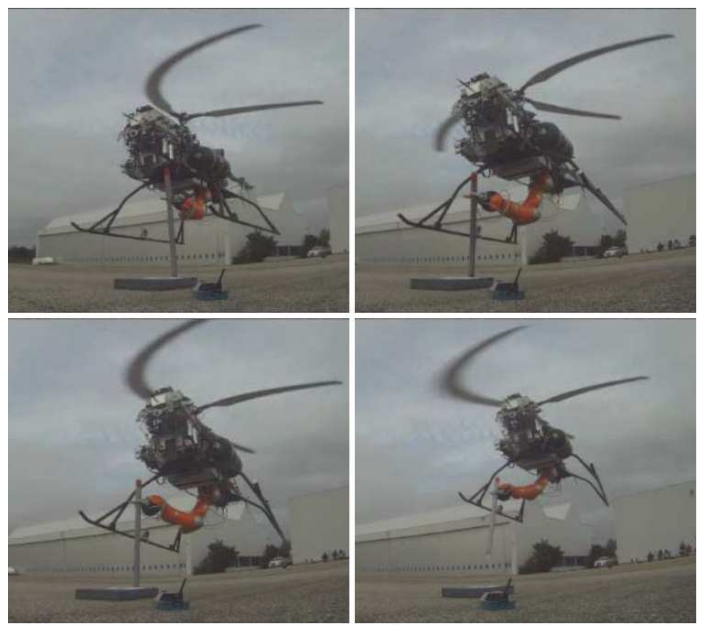

Fig. 13. This sequence shows the robot arm compensating the helicopter movement during the grasping task.

\section{CONCLUSIONS}

In this paper we presented a first system for aerial manipulation composed of a helicopter and a fully actuated redundant robot arm. The coupling between helicopter and moving arm generates interesting 'hidden' effects. One of them - the low frequency diverging oscillations (phase circles) - we described in the paper. We have shown that the phase circles are eliminated by restricting the movement of arm CoG in the lateral plane of the helicopter. Utilizing this result we proposed the control approach for the whole system. The proposed controller is composed of the helicopter position controller and arm controller with mentioned motion restriction for the arm $\mathrm{CoG}$. This restriction constitutes the coupling between two controllers on kinematical level. Using a 7-DoF arm, we are able to move TCP with 6-DoF - without drawbacks for manipulation tasks.

In flight experiments we have shown that a system with a serial manipulator mounted on the helicopter fuselage could be operated. Please note that a theoretical proof of this fact or a reliable computer simulation is hardly possible. We have also shown in flight experiments that using the proposed controller a simple manipulation is possible. We think that for many practical applications the usage of a fully actuated arm with a payload of about $10 \mathrm{~kg}$ is required. So the setup we presented in this paper is a starting point for practical investigations of these applications and for developing of corresponding technologies. The next steps in our research work will be devoted to increasing the TCP positioning precision and to investigations of force interaction between TCP and object. The mechanical mounting of the arm will be also modified in order to increase the workspace of TCP.

\section{ACKNOWLEDGMENT}

This work has been partially founded by the European Commission's Seventh Framework Programme as part of the project ARCAS (ICT-287617) and the DLR Helman Project.

\section{REFERENCES}

[1] K. Kondak, A. Ollero, I. Maza, K. Krieger, A. Albu-Schäffer, M. Schwarzbach, and M. Laiacker, Handbook of Unmanned Aerial Vehicles. Berlin, Germany: Springer-Verlag, 2013, ch. Unmanned Aerial Systems Physically Interacting with the Environment. Load Transportation, Deployment and Aerial Manipulation.

[2] A. Albers, S. Trautmann, T. Howard, T. A. Nguyen, M. Frietsch, and C. Sauter, "Semiautonomous flying robot for physical interaction with environment," in Robotics, Automation and Mechatronics, 2010.

[3] P. Pounds and A. Dollar, "Hovering stability of helicopters with elastic constraints," in Proceedings of the ASME Dynamic Systems and Control Conference, 2010.

[4] P. E. I. Pounds, D. R. Bersak, and A. M. Dollar, "Grasping from the air: Hovering capture and load stability," in ICRA. IEEE, 2011, pp. 2491-2498.

[5] D. Mellinger, M. Shomin, N. Michael, and V. Kumar, "Cooperative grasping and transport using multiple quadrotors," in Proceedings of the International Symposium on Distributed Autonomous Robotic Systems, November 2010.

[6] D. Mellinger, Q. Lindsey, M. Shomin, and V. Kumar, "Design, modeling, estimation and control for aerial grasping and manipulation," in IROS. IEEE, 2011, pp. 2668-2673.

[7] C. M. Korpela, T. W. Danko, and P. Y. Oh, "MM-UAV: Mobile manipulating unmanned aerial vehicle," Journal of Intelligent and Robotic Systems, vol. 65, no. 1-4, pp. 93-101, 2012.

[8] S. Abiko, R. Lampariello, and G. Hirzinger, "Impedance control for a free-floating robot in the grasping of a tumbling target with parameter uncertainty," in Intelligent Robots and Systems, 2006 IEEE/RSJ International Conference on, oct. 2006, pp. $1020-1025$.

[9] K. Kondak, M. Bernard, N. Losse, and G. Hommel, "Elaborated modeling and control for autonomous small size helicopters," in International ISR/Robotik Joint Conference. VDI, 2006, pp. 207208.

[10] K. Kondak, M. Bernard, N. Meyer, and G. Hommel., "Autonomously flying vtol-robots: Modeling and control." IEEE International Conference on Robotics and Automation, ICRA, 2007.

[11] K. Kondak, K. Krieger, A. Albu-Schäffer, M. Schwarzbach, M. Laiacker, I. Maza, A. Rodriguez-Castano, and A. Ollero, "Closed-loop behavior of an autonomous helicopter equipped with a robotic arm for aerial manipulation tasks." IJARS, 2013.

[12] A. Albu-Schäffer, C. Ott, and G. Hirzinger, "A unified passivitybased control framework for position, torque and impedance control of flexible joint robots," The International Journal of Robotics Research, vol. 26, no. 1, pp. 23-39, 2007.

[13] C. Ott, Cartesian Impedance Control of Redundant and Flexible-Joint Robots. Berlin, Germany: Springer-Verlag, 2008.

[14] S. Parusel, S. Haddadin, and A. Albu-Schäffer, "Modular state-based behavior control for safe human-robot interaction: A lightweight control architecture for a lightweight robot," in IEEE International Conference on Robotics and Automation, ICRA 2011,, 2011, pp. 42984305 . 